\title{
High efficacy of third generation EGFR inhibitor AZD9291 in a leptomeningeal carcinomatosis model with EGFR-mutant lung cancer cells
}

\author{
Shigeki Nanjo ${ }^{1}$, Hiromichi Ebi ${ }^{1}$, Sachiko Arai ${ }^{1}$, Shinji Takeuchi ${ }^{1}$, Tadaaki Yamada $^{1}$, \\ Satsuki Mochizuki², Yasunori Okada², Mitsutoshi Nakada ${ }^{3}$, Takashi Murakami ${ }^{4}$, \\ Seiji Yano ${ }^{1}$ \\ ${ }^{1}$ Department of Medical Oncology, Kanazawa University Cancer Research Institute, Kanazawa, Japan \\ ${ }^{2}$ Department of Pathology, Keio University School of Medicine, Tokyo, Japan \\ ${ }^{3}$ Department of Neurosurgery, Graduate School of Medical Science, Kanazawa University, Kanazawa, Japan \\ ${ }^{4}$ Laboratory of Tumor Biology, Takasaki University of Health and Welfare, Takasaki, Japan \\ Correspondence to: Seiji Yano, e-mail: syano@staff.kanazawa-u.ac.jp
}

Keywords: leptomeningeal carcinomatosis, EGFR-TKI resistance, EGFR mutation, EGFR inhibitors

Received: August 06, 2015

Accepted: November 29, 2015

Published: December 24, 2015

\section{ABSTRACT}

Leptomeningeal carcinomatosis (LMC) remarkably decreases the quality of life of EGFR-mutant lung cancer patients. In contrast to the lesions outside the central nervous system (CNS), molecular mechanisms of EGFR tyrosine kinase inhibitor (TKI) resistance in CNS lesions including LMC are largely unknown. In this study, we established an in vivo imaging model for LMC with EGFR mutant lung cancer cell lines harboring an exon 19 deletion in EGFR and evaluated the effect of first generation EGFR-TKIs, erlotinib, second generation afatinib, and third generation AZD9291. In PC-9/ffluc model, erlotinib treatment slowed the development of LMC. Importantly, treatment with afatinib or AZD9291 apparently delayed the development of LMC. Moreover, treatment with a higher dose of AZD9291, also associated with inhibited phosphorylation of EGFR downstream molecule S6, regressed LMC refractory to the aforementioned EGFR-TKI treatments. These observations suggest that the third generation EGFR-TKI AZD9291 may be an effective treatment for first or second generation EGFR-TKI resistant LMC caused by EGFR-mutant lung cancer.

\section{INTRODUCTION}

Central nervous system (CNS) metastasis including brain metastasis and leptomeningeal carcinomatosis (LMC) often develops in several types of cancers such as lung, breast, and renal cancer. It is a grave complication that shortens survival and markedly diminishes the quality of life of the patients [1]. The epidermal growth factor receptor (EGFR)-mutant-lung cancer represents $10 \%$ and $25 \%$ of non-small-cell lung cancers (NSCLC) in Caucasians and East Asians, respectively [2]. LMC develops in patients that have the EGFR mutation more often compared with patients without the mutation [3]. EGFR-tyrosine kinase inhibitors (EGFR-TKIs) such as first generation gefitinib/erlotinib and second generation afatinib showed remarkable activity in EGFR-mutant lung cancer patients with or without CNS metastasis $[4,5,6]$. However, EGFR-TKI resistance may eventually develop after varying periods of treatment, and approximately onethird of patients develop CNS metastases after acquisition of EGFR-TKI resistance [7-9]. Brain metastases are manageable by concomitant use of EGFR-TKI and radiation therapy including whole brain irradiation and stereotactic radiotherapy [10]. There is, however, no established therapy for LMC, which is resistant to first and second generation EGFR-TKIs. Therefore, novel and effective therapies need to be developed for managing LMC in cancer patients.

Third generation EGFR-TKIs such as AZD9291 and CO-1686 effectively block EGFRs with the T790M mutation as well as exon 19 deletion and L858R-sensitive mutations, but do not inhibit wild type EGFRs. They have also shown clinical efficacy in EGFR-mutant lung cancer patients who previously treated with EGFR-TKIs [11, 12]. However, the efficacy of third generation EGFR-TKIs on CNS metastasis is not well reported in literature. 
In the present study, we established an in vivo imaging model for LMC with an EGFR-TKI sensitive EGFR-mutant lung cancer cell lines. Furthermore, we evaluated the efficacy of AZD9291 in comparison with erlotinib and afatinib in our LMC model.

\section{RESULTS}

\section{Establishment of an in vivo imaging model for LMC of EGFR-mutant lung cancer}

To establish an LMC model with an EGFRmutant lung cancer, we utilized the human EGFR mutant lung adenocarcinoma PC-9/ffluc (exon 19 deletion) [13], HCC827/luc, and H1975/luc cells, which were transfected with the fusion gene of luciferase for in vivo imaging. EGFR-TKIs such as gefitinib, erlotinib, afatinib, and AZD9291 decreased viability of PC-9/ ffluc and HCC827/luc cells (Figure 1A, Supplementary Figure 4). On the other hand, only afatinib and AZD9291 decreased viability of H1975/luc cells (Supplementary Figure 1). There was no discernible difference between luciferase-gene transfectants and parental cells, in terms of sensitivity to EGFR-TKIs. Western blot analysis of PC-9/ ffluc revealed that AZD9291 inhibited phosphorylation of EGFR and its downstream molecule $\mathrm{S} 6$ in a dosedependent manner (Figure 1B). We inoculated PC-9/ ffluc cells into the leptomeningeal space of SHO-SCID mice (Figure 2A). PC-9/ffluc cells (more than $1.6 \times 10^{3}$ ) developed LMC in SHO-SCID mice. The survival of the recipient mice was shortened in a cell numberdependent manner (Figure 2B). All mice inoculated with $2 \times 10^{5}$ PC-9/ffluc cells developed LMC (Figure 2C, 2D) and became moribund within 28 days; we used this experimental protocol for the following experiments.

\section{Effect of EGFR-TKIs in the LMC model}

We sought to examine the effect of AZD9291 in our LMC model, as compared with erlotinib. In preliminary experiments with the subcutaneous tumor model, daily oral treatment with $25 \mathrm{mg} / \mathrm{kg}$ of erlotinib remarkably prevented the enlargement of PC-9/ffluc tumors for more than 23 days. In the same subcutaneous model, daily oral treatment with $6 \mathrm{mg} / \mathrm{kg}$ of AZD9291 also prevented the enlargement of PC-9/ffluc tumors (Figure 3A). Therefore, we determined that $25 \mathrm{mg} / \mathrm{kg}$ of erlotinib and $6 \mathrm{mg} / \mathrm{kg}$ of AZD9291 have equivalent activity in our mouse model.

In the LMC model with PC-9/ffluc cells, the mice in the control group became moribund within 28 days after tumor cell inoculation. Daily erlotinib treatment $(25 \mathrm{mg} / \mathrm{kg}$ ) remarkably delayed the progression of LMC, indicating that this dose of erlotinib was effective against LMC, which is consistent with its reported clinical activity [15]. Importantly, daily oral treatment with $6 \mathrm{mg} / \mathrm{kg}$ of AZD9291 further delayed the progression of LMC
(Figure 3B, 3C). In parallel experiments, HCC827/luc and H1975/luc cells developed LMC, and AZD9291 showed efficacy against the LMC produced by HCC827/luc and H1975/luc cells (Figure 4). These observations clearly indicate that in addition to erlotinib, AZD9291 has activity against LMC of EGFR-mutant lung cancer.

\section{Effect of high dose of AZD9291 on LMC after acquiring EGFR-TKI resistance}

We next sought to examine whether a higher dose of AZD9291 showed activity against an erlotinib-resistant LMC model. When the mice with progressed LMC by PC-9/ffluc cells after continuous treatment with $25 \mathrm{mg} /$ $\mathrm{kg}$ of erlotinib were treated with a high dose of AZD9291 (25 $\mathrm{mg} / \mathrm{kg}$ ), the LMC was regressed (Figure 5A). The high dose-AZD9291 treatment also regressed LMC that had progressed after treatment with $6 \mathrm{mg} / \mathrm{kg}$ of AZD9291 (Figure 5A). Interestingly, phosphorylated S6 in leptomeningeal cancer cells was suppressed after a high dose of AZD9291, as determined by immunofluorescence analysis (Figure 5B). This confirmed the efficacy of high dose-AZD9291 treatment against LMC. In parallel experiments, we obtained similar results with LMC models treated with afatinib. Briefly, $5 \mathrm{mg} / \mathrm{kg}$ of afatinib, which could prevent the enlargement of subcutaneous PC-9/ffluc tumors (Figure 6A), slowed the progression of LMC. However, after continuous treatment with afatinib, LMC in the treated mice progressed. It should be noted that the high dose of ADZ9291 (25 mg/ $\mathrm{kg}$ ) could regress LMC refractory to afatinib treatment (Figure 6B).

\section{Toxicity profiles of EGFR-TKIs in vivo}

To evaluate the toxicity of EGFR-TKI treatment, we measured the body weights of the mice during treatment. Treatment with EGFR-TKIs including administration of a high dose of AZD9291 did not caused severe body weight loss (Supplementary Figures 2, 3, 4). Furthermore, we assessed skin damage because AZD9291 has been reported to have less activity against wild type EGFR compared with mutant EGFRs such as exon 19 deletion, and L858R and T790M [15]. Accordingly, expression of phosphorylated mitogen-activated protein kinase (pMAPK), a downstream molecule of EGFR in normal skin, remained present after continuous treatment with $6 \mathrm{mg} / \mathrm{kg}$ of AZD9291, while it was inhibited by the continuous treatment with $25 \mathrm{mg} / \mathrm{kg}$ of erlotinib or $5 \mathrm{mg} / \mathrm{kg}$ of afatinib (Figure 7). Moreover, inhibition of EGFR phosphorylation due to treatment with erlotinib or afatinib could be reversed after continuous treatment with $25 \mathrm{mg} / \mathrm{kg}$ of AZD9291. These results strongly suggest that AZD9291 at the dose without wild-type EGFR inhibition is tolerable and sufficient for controlling LMC refractory to first and second generation EGFR-TKIs. 


\section{DISCUSSION}

In the present study, we have established an in vivo imaging LMC model of EGFR-mutant lung cancer and demonstrated that third generation EGFR-TKI, AZD9291, can manage LMC in our model.

A recent study reported promising results with AZD9291 treatment (response rate of 51\%, and progression free survival of 8.2 months) in patients who had experienced radiographic progression after administration of at least one EGFR-TKI [11]. However, since the effect of AZD9291 specifically on CNS lesions including LMCs was not reported in the study, the activity of AZD9291 against LMC was unknown. Here, we clearly showed that AZD9291 has potential against EGFR-TKI naïve LMC of EGFR-mutant lung cancer cells. In addition, AZD9291 may also have activity against LMC which is resistant to first and second generation EGFR-TKIs. Based on our preclinical evidence, we posit that clinical trials are required to evaluate the efficacy of AZD9291 treatment in EGFRmutant lung cancer patients with CNS metastasis including LMC with or without resistance to other EGFR-TKIs.

The ineffectiveness of EGFR-TKIs on CNS metastasis is thought to be due to insufficient penetration of EGFRTKIs [5]. This is corroborated by the evidence that shows erlotinib, which has better penetration in CNS lesions

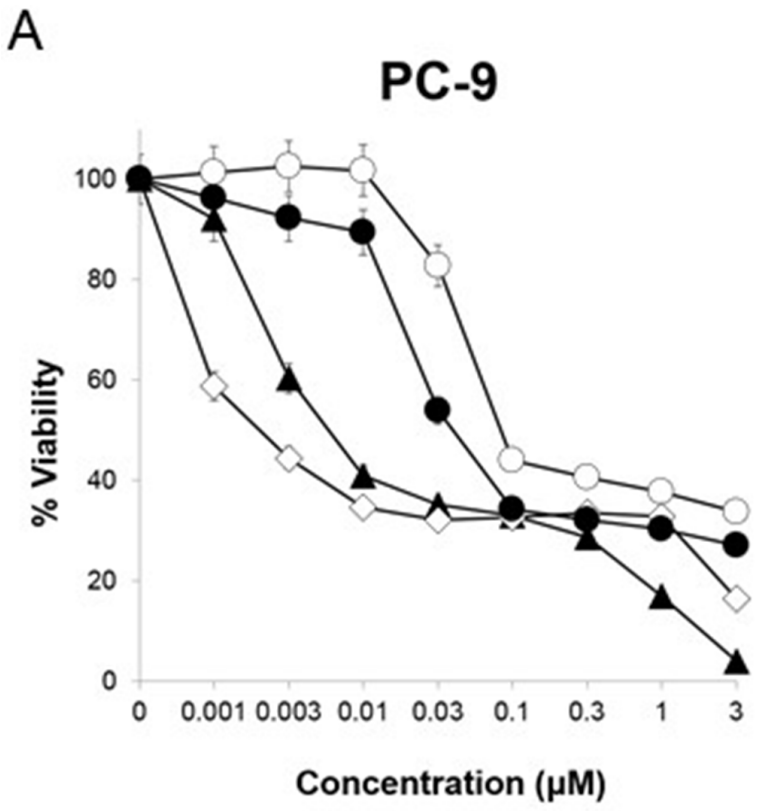

\section{PC-9/ffluc}

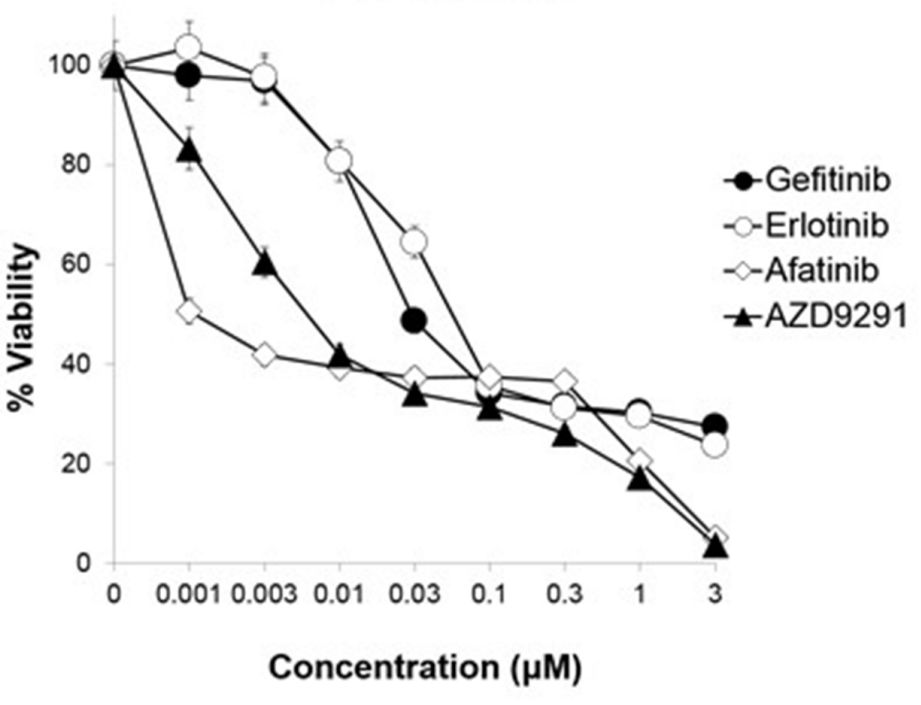

B PC-9/ffluc

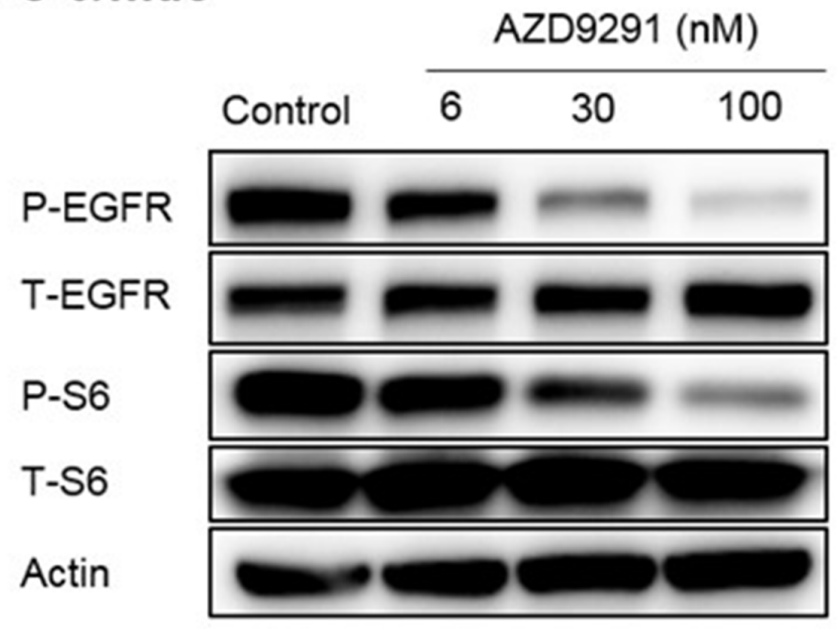

Figure 1: Sensitivity of PC-9/ffluc cells to EGFR-TKIs in vitro. (A) PC-9, and PC-9/ffluc cells $\left(2 \times 10^{3}\right.$ cells per well) were incubated with various concentrations of erlotinib, gefitinib, AZD9291, and afatinib for 72 hours. Cell growth was determined by the MTT assay. Bars represent SD. (B) PC-9/ffluc cells were incubated with AZD9291 (6, 30, $100 \mathrm{nmol} / \mathrm{L})$ for 24 hours. The cell lysates were harvested and phosphorylation of indicated proteins was determined by western blot analysis. Data shown are representative of three independent experiments with similar results. 


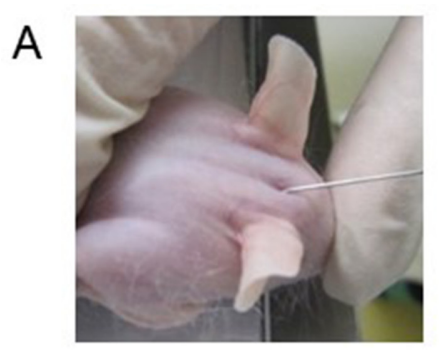

\begin{tabular}{ccc}
\hline No. of cells & Incidence & MST (days) \\
\hline $1.6 \times 10^{3}$ & $2 / 4(50 \%)$ & N.R. \\
$8 \times 10^{3}$ & $4 / 4(100 \%)$ & N.R. \\
$4 \times 10^{4}$ & $4 / 4(100 \%)$ & 48 \\
$2 \times 10^{5}$ & $4 / 4(100 \%)$ & 28 \\
\hline
\end{tabular}
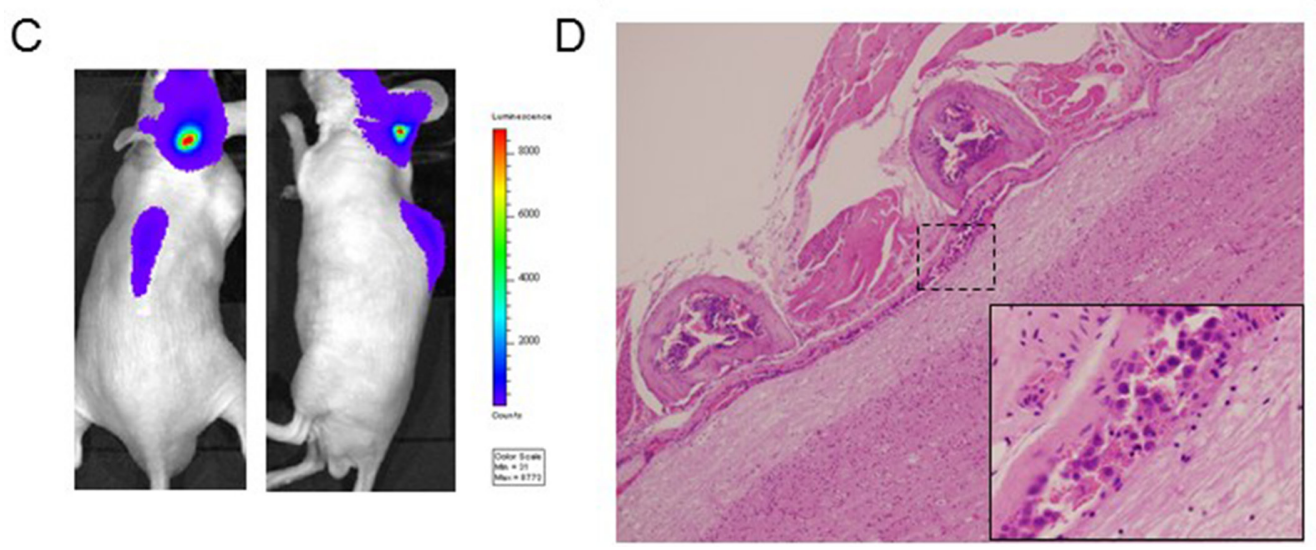

Figure 2: Leptomeningeal carcinomatosis model with PC-9/ffluc cells. (A) PC-9/ffluc cells were inoculated into the space between the external occipital protuberance and first cervical vertebra of SHO-SCID mice. (B) Production of LMC was determined using the IVIS imaging system; MST: median survival time; N.R: not reached. (C) Representative imaging of mice immediately after PC-9/ffluc cell inoculation. (D) Representative histological images of spinal cord with LMC ( $\mathrm{x} 40)$. The high-power field image of dotted area is shown in the lower right corner $(\mathrm{x} 400)$.

A

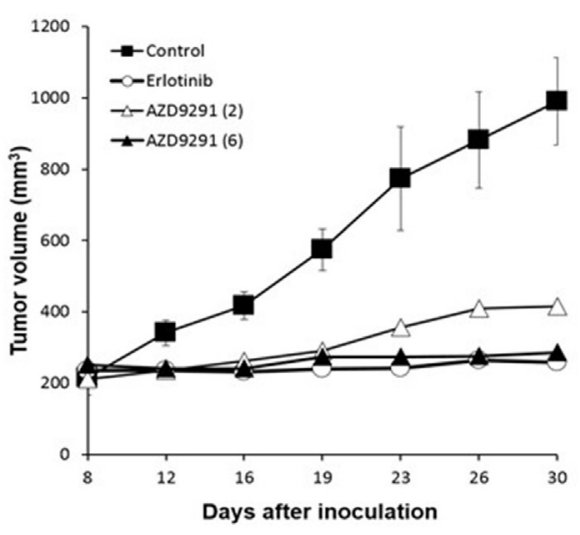

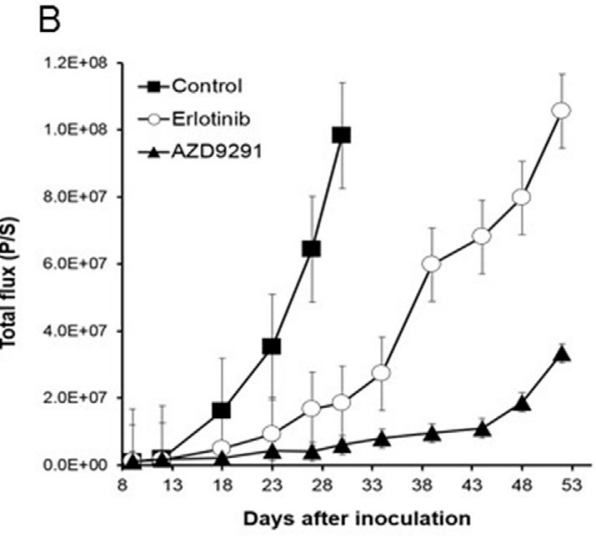

C

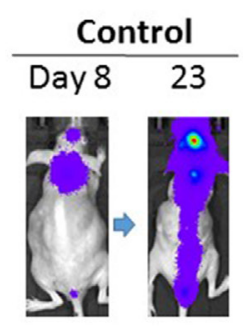

Erlotinib

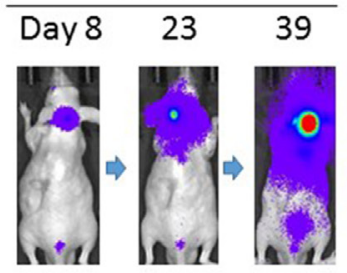

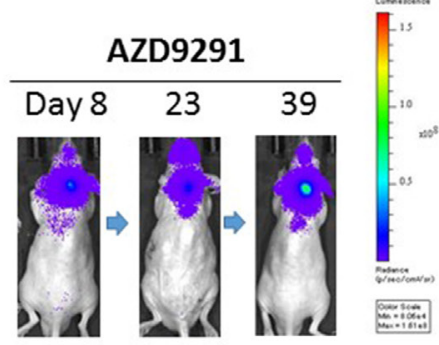

Figure 3: Effect of EGFR-TKI treatment in subcutaneous and LMC models with PC-9/ffluc cells. (A) PC-9/ffluc cells were inoculated subcutaneously into SHO-SCID mice $(N=4)$. Daily oral treatment with erlotinib $(25 \mathrm{mg} / \mathrm{kg})$ or AZD9291 $(6 \mathrm{mg} / \mathrm{kg}) \mathrm{was}$ given from day 8 until day 30. (B) PC-9/ffluc cells were inoculated into the leptomeningeal space of SHO-SCID mice $(N=5)$. Daily oral treatment with erlotinib $(25 \mathrm{mg} / \mathrm{kg}$ ) or AZD9291 $(6 \mathrm{mg} / \mathrm{kg})$ was given from day 5 until day 50 . Bars represent SD. (C) Representative images of mice treated with or without erlotinib $(25 \mathrm{mg} / \mathrm{kg})$ or AZD9291 $(6 \mathrm{mg} / \mathrm{kg})$. 
than gefitinib, more effectively treats CNS metastasis resistant to gefitinib treatment [14, 16]. However, gefitinib is generally effective against EGFR-TKI naïve CNS metastasis in EGFR-mutant lung cancer patients, even though only $1 \%$ of gefitinib penetrates into the CNS lesions [4, 5, 16, 17]. These observations suggest the involvement of other mechanisms that result in the development of marginal resistance to a low concentration of EGFR-TKIs in CNS lesions. Unfortunately, we could not recover tumor cells from the LMC model. Thus, the molecular mechanism by which PC-9/ffluc cells acquire resistance to EGFR-TKI treatment remains unknown.

For LMC resistant to the daily standard EGFRTKI treatment, no effective therapy has been established. Intermittent high dose EGFR-TKI and EGFR-TKI
A

\begin{tabular}{cccc}
\hline Cell line & Incidence & MST (days) \\
\hline HCC827/luc & $7 / 9 \quad(78 \%)$ & $>40$ \\
H1975/luc & $6 / 6(100 \%)$ & 24 \\
\hline
\end{tabular}

C

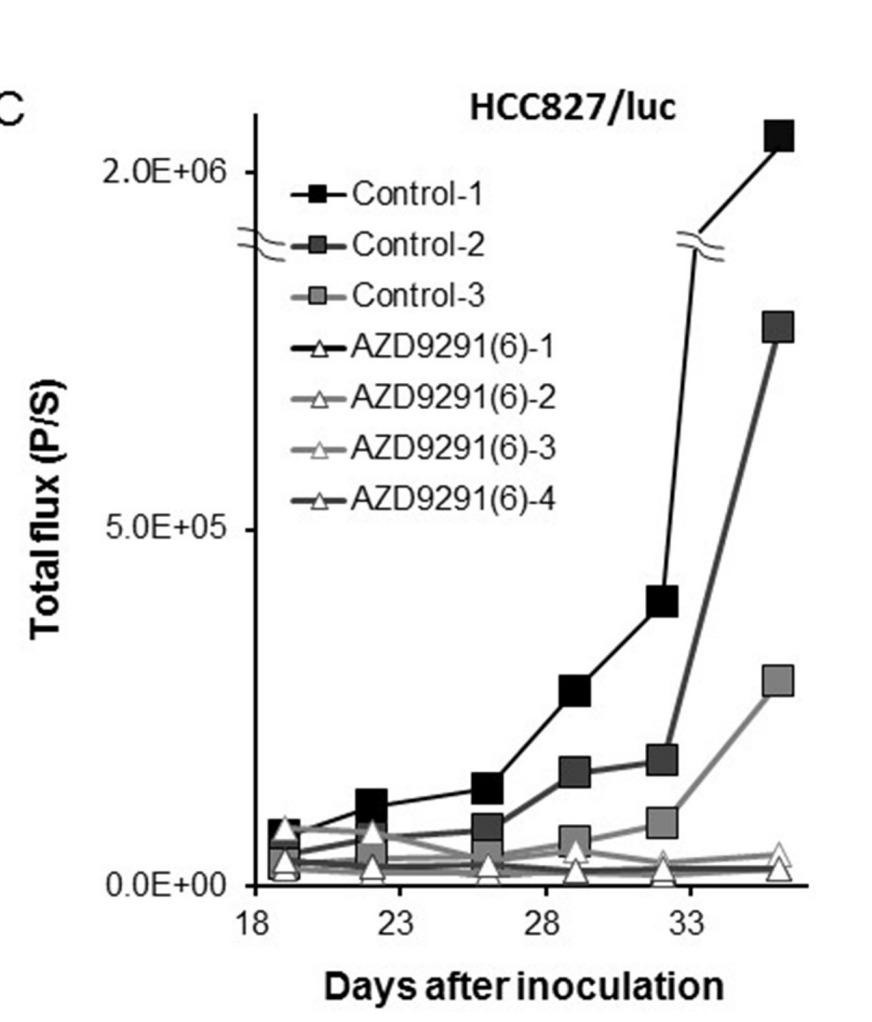

pulse therapy was proposed previously [18]. However, recent retrospective studies reported that there was no significant difference between the effect of a daily standard dose of erlotinib and high dose erlotinib pulse therapy on the overall survival of patients $[19,20]$. We also examined the effect of gefitinib-pulse treatment in our LMC model. However, gefitinib-pulse treatment was not superior to daily gefitinib treatment in the control (Supplementary Figure 5).

Afatinib is an irreversible EGFR-TKI that has been approved in many countries including US, EU, and Japan. The results of the compassionate-use program of afatinib indicate that this drug is equally effective in EGFR-mutant lung cancer patients with CNS metastasis compared with the patients without CNS metastasis

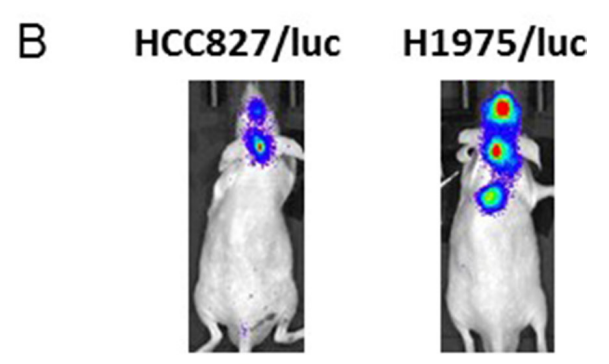

D

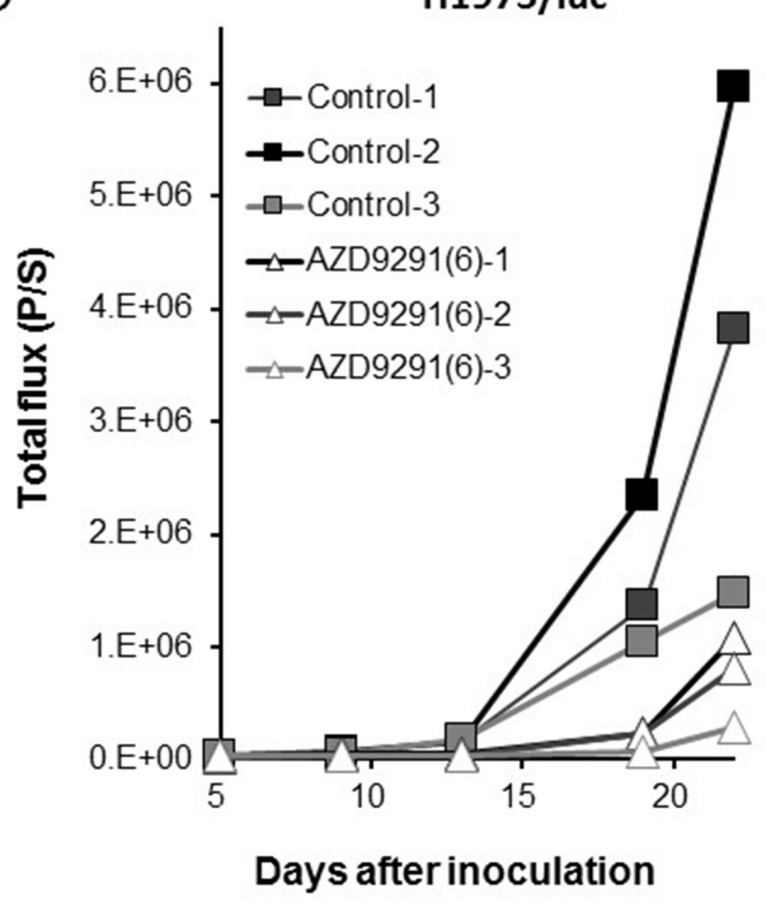

Figure 4: Effect of EGFR-TKI treatment in LMC models with HCC827/luc and H1975/luc cells. HCC827/luc and H1975/luc cells were inoculated into the space between the external occipital protuberance and first cervical vertebra of SHO-SCID mice. $(\mathbf{A}, \mathbf{B})$ Production of LMC was determined using the IVIS imaging system; MST: median survival time. (C) The mice inoculated with HCC827/luc cells were daily treated with control $(N=3)$ or AZD9291 $(6 \mathrm{mg} / \mathrm{kg})(N=4)$ from day 18 until day 36 . (D) The mice inoculated with H1975/luc cells were daily treated with control $(N=3)$ or AZD9291 $(6 \mathrm{mg} / \mathrm{kg})(N=3)$ from Daily oral treatment with AZD9291 $(6 \mathrm{mg} / \mathrm{kg})$ was given from day 5 until day 22. 
[7]. We found that afatinib at a dose of $5 \mathrm{mg} / \mathrm{kg}$, which was almost equivalent to $25 \mathrm{mg} / \mathrm{kg}$ of erlotinib against subcutaneous tumors with PC-9/ffluc cells, remarkably delayed the progression of LMC in our model (Figure 5). The clinical efficacy of afatinib on CNS metastasis could be replicated in the LMC model, confirming clinical relevance of this model. Therefore, our LMC model may be useful for testing experimental and novel therapies for LMC.

In conclusion, we have established an LMC model for EGFR-mutant lung cancer and demonstrated the potential of third generation EGFR-TKI AZD9291, on not
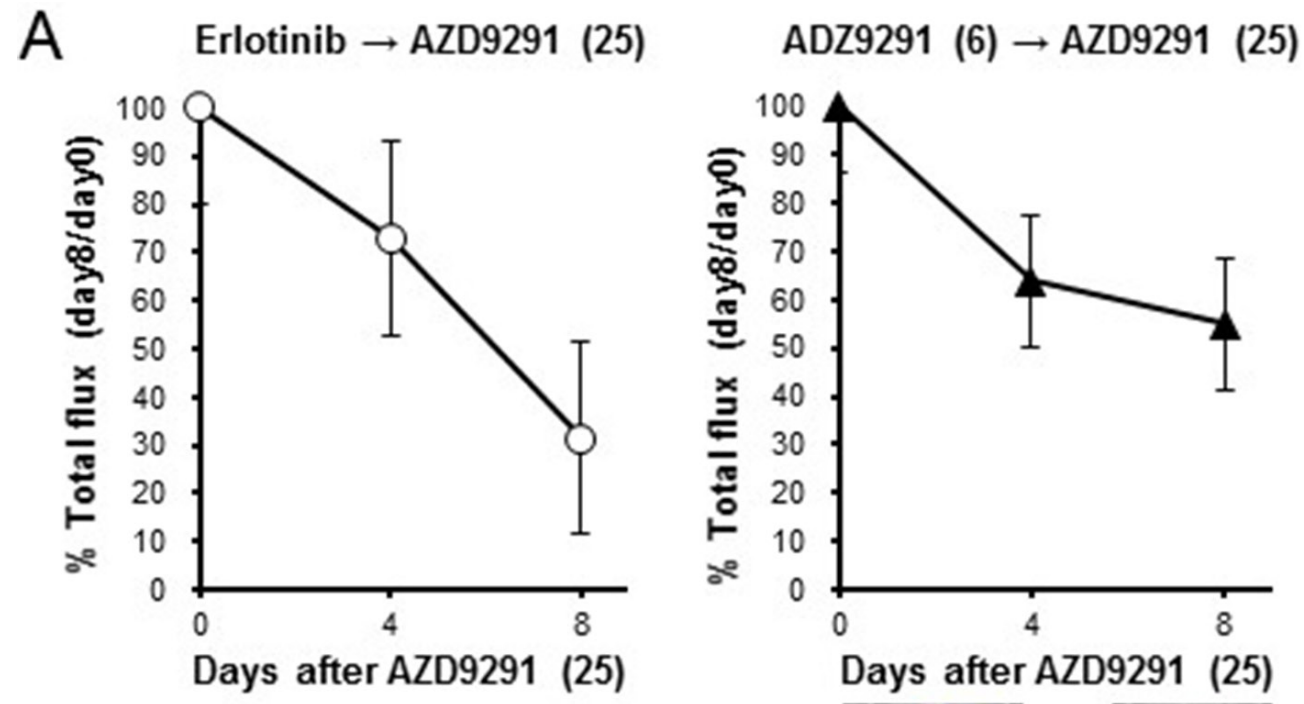

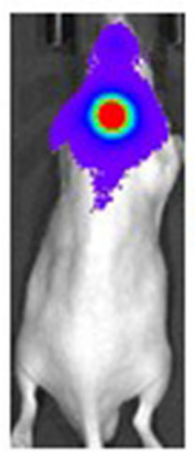

Day 0

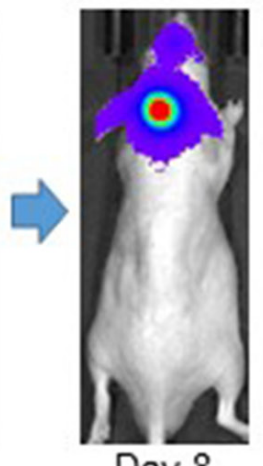

Day 8
After Erlotininb

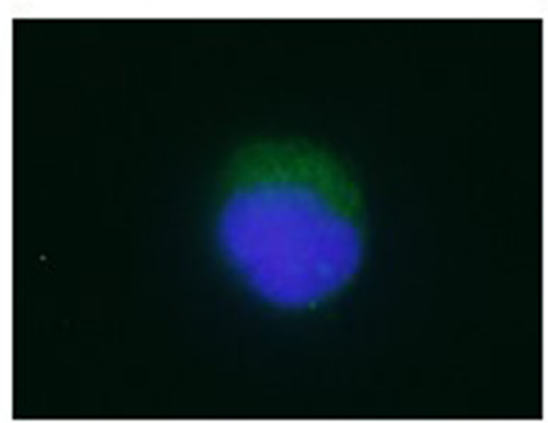

Days after AZD9291 (25)

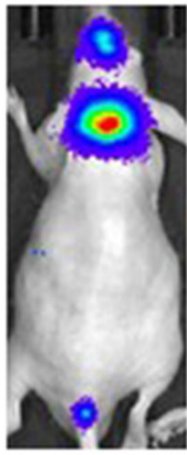

Day 0

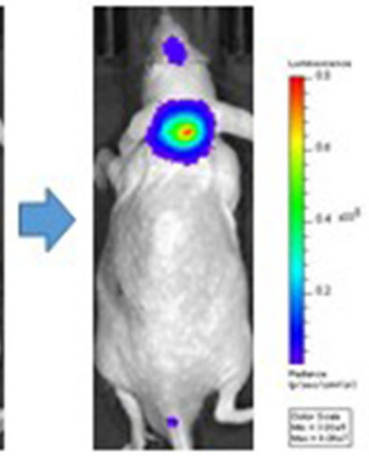

Day 8
After AZD9291 (25)

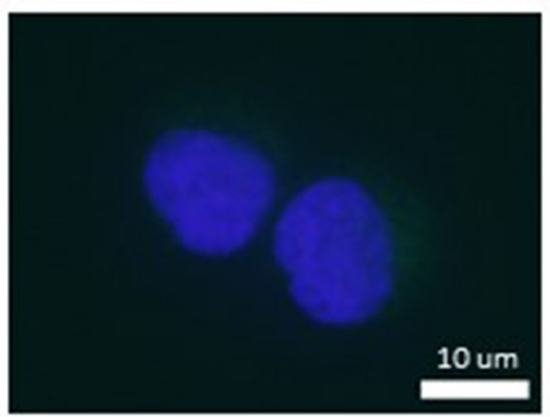

Figure 5: Effect of high dose-AZD9291 treatment after acquisition of EGFR-TKI resistance. (A) After acquisition of resistance to treatment with $25 \mathrm{mg} / \mathrm{kg}$ of erlotinib $(N=5)$ or $6 \mathrm{mg} / \mathrm{kg}$ of AZD9291 $(N=5)$, the mice were given daily oral treatment with AZD9291 $(25 \mathrm{mg} / \mathrm{kg})$ for 8 days. The representative images are shown. (B) Cancer cells from the leptomeningeal space were collected after development of erlotinib resistance and high dose-AZD9291 treatment. The cell pellets were subsequently analyzed for S6 phosphorylation by immunofluorescence. 

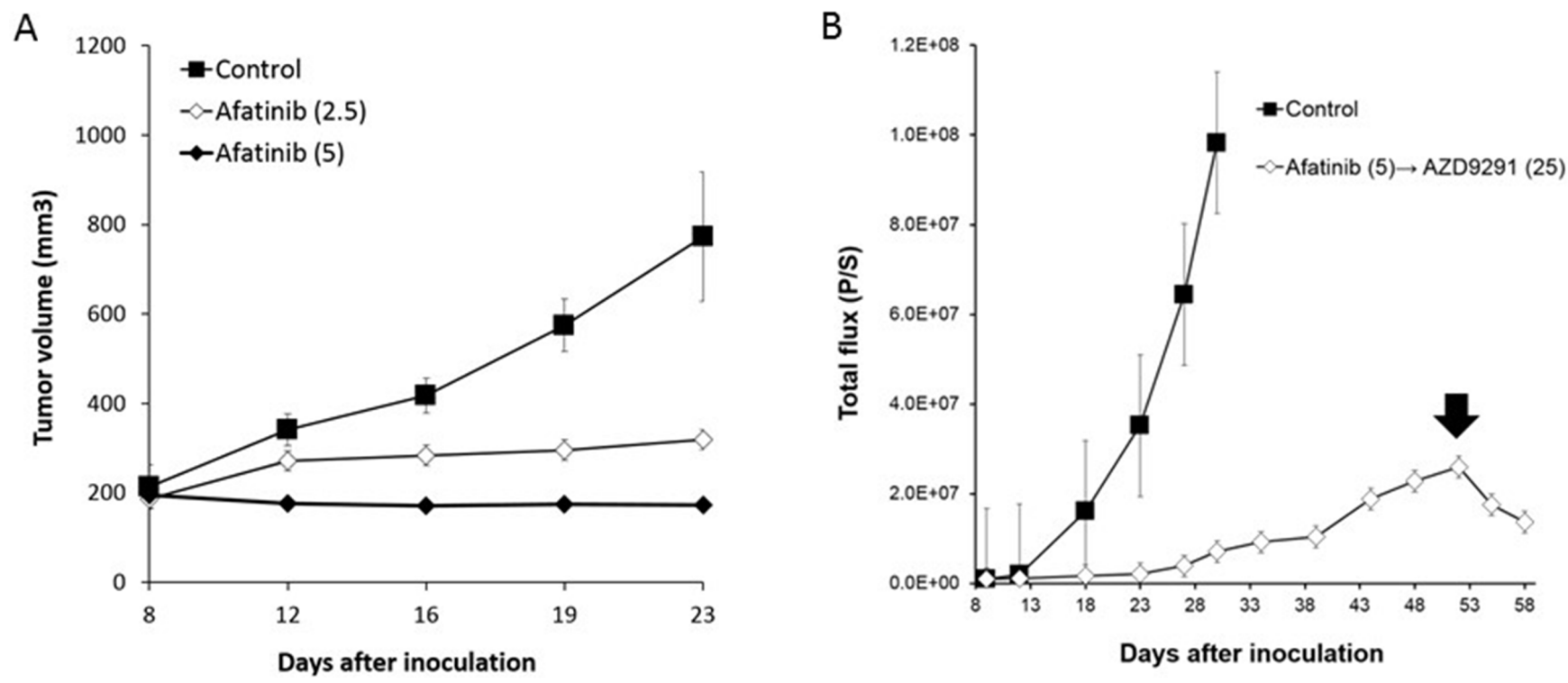

Figure 6: The efficacy of afatinib and high dose-AZD9291 after afatinib resistance in LMC model. (A) PC-9/ffluc cells were inoculated subcutaneously in SHO-SCID mice. Mice were treated with $2.5 \mathrm{mg} / \mathrm{kg}(N=5) \mathrm{or} 5 \mathrm{mg} / \mathrm{kg}$ of afatinib $(N=5)$. Treatment was given daily for 23 days. Tumor volume was measured using calipers on the indicated days. Mean $\pm \mathrm{SE}$ tumor volumes are shown. (B) LMC mice with PC-9/ffluc cells were administered afatinib $(5 \mathrm{mg} / \mathrm{kg})$ daily from day 8 until day 50 . Luminescence was evaluated as total flux (p/s: photons/second). After resistance was acquired to afatinib, the LMC mice were administered AZD9291 (25 mg/kg) once daily for 8 days (from day 51 until day 58). Arrow indicates the initiation of the high dose AZD9291 (25 mg/kg) treatment.

Control

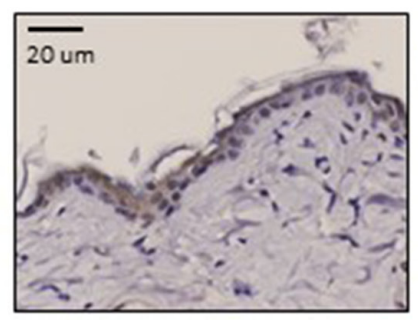

AZD9291 (25)
Erlotinib

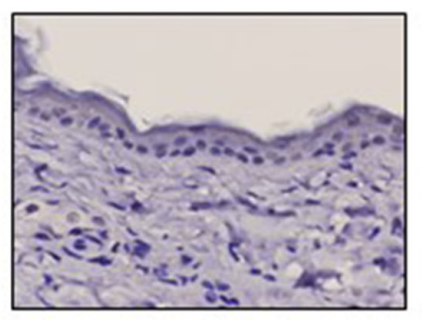

$\sqrt{5}$

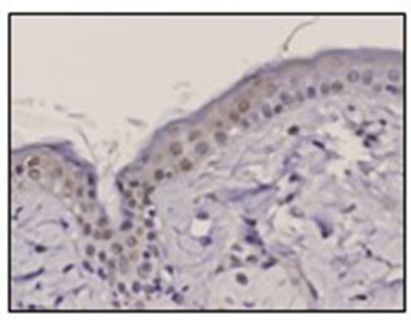

Afatinib

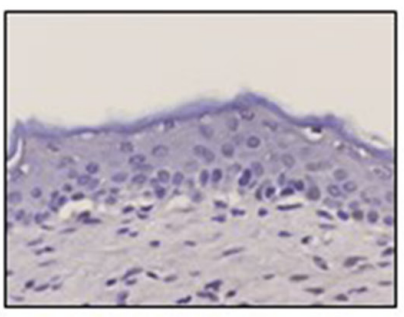

ת

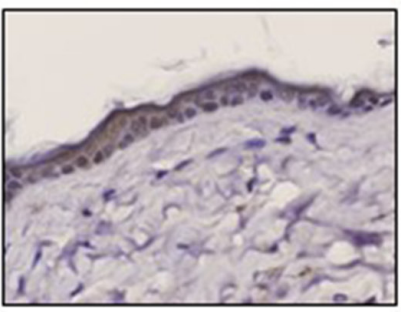

ADZ9291 (6)
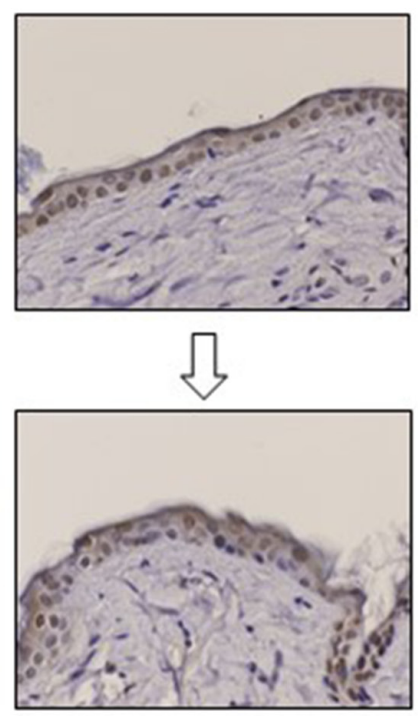

Figure 7: The effect of continuous EGFR-TKI treatment on MAPK phosphorylation in normal skin cells. PC-9/ffluc cells were inoculated subcutaneously in SHO-SCID mice. Mice were treated daily with $25 \mathrm{mg} / \mathrm{kg}$ of erlotinib, $5 \mathrm{mg} / \mathrm{kg}$ of afatinib, or $6 \mathrm{mg} / \mathrm{kg}$ of AZD9291 for 50 days. Then, small skin tissues $(3 \mathrm{~mm} \times 3 \mathrm{~mm})$ were harvested from the mice (upper panels). The mice were further treated with $25 \mathrm{mg} / \mathrm{kg}$ of AZD9291 for 8 days, and then small skin tissues $(3 \mathrm{~mm} \times 3 \mathrm{~mm}$ ) were harvested again from them (lower panels). Phosphorylated ERK expression in these skin lesions was evaluated by immunohistochemistry. (x400). 
only EGFR-TKI naïve LMC but also EGFR-TKI-refractory LMC. Our preclinical evidence suggest therapeutic potential of AZD9291 on EGFR-mutant lung cancer patients with CNS metastasis including LMC with or without resistance to other EGFR-TKIs.

\section{MATERIALS AND METHODS}

\section{Cell cultures and reagents}

The EGFR-mutant human lung adenocarcinoma cell line PC-9 with deletions in EGFR exon 19 (del E746 A750) and HCC827, with deletions in EGFR exon 19 were purchased from Immuno-Biological Laboratories Co. (Gunma, Japan) and the American Type Culture Collection (Manassas, VA), respectively. H1975 cells, with the L858R/T790M double mutations in $E G F R$, were kindly provided from Drs. Yoshitaka Sekido (Aichi Cancer Center Research Institute, Japan) and John D. Minna (University of Texas Southwestern Medical Center). H1975/luc (JCRB1486) and HCC827/ luc (JCRB1516) cells, which are luciferase transfected by Dr. Takashi Murakami (Laboratory of Tumor Biology, Takasaki University of Health and Welfare, Takasaki, Gunma, Japan), were provided from JCRB cell Bank (Osaka, Japan). PC-9 ffLuc-cp156 transfectant (PC-9/ ffluc) was established as previously described [13]. These cells were maintained in RPMI-1640 medium supplemented with $10 \%$ fetal bovine serum (FBS) and antibiotics. All cells were passaged for less than 3 months before renewing frozen, early-passage stocks. Cells were regularly screened for mycoplasma contamination using MycoAlert Mycoplasma Detection Kits (Lonza, Rockland, $\mathrm{ME})$. The cell lines were authenticated at the laboratory of the National Institute of Biomedical Innovation (Osaka, Japan) by short tandem repeat analysis in May 2015. Gefitinib, erlotinib, afatinib, and AZD9291 were obtained from Selleck Chemicals (Houston, TX).

\section{Cell viability assay}

Cell viability was measured by the MTT dye reduction method. Tumor cells were plated at a density of $2 \times 10^{3} / 100 \mu \mathrm{l}$ per well in RPMI 1640 plus $10 \%$ FBS in 96-well plates and incubated for 24 hours. EGFRTKIs were then added to each well, and incubation was continued for another 72 hours. Cell growth was measured with MTT solution (2 mg/mL; Sigma, St. Louis, MO), as described [21].

\section{Western blot}

Protein aliquots of $25 \mu \mathrm{g}$ each were resolved by SDS polyacrylamide gel (Bio-Rad, Hercules, CA) electrophoresis and transferred to polyvinylidene difluoride membranes (Bio-Rad). After washing thrice, the membranes were incubated with Blocking One (Nacalai Tesque, Inc., Kyoto, Japan) for 1 hour at room temperature and then incubated overnight at $4{ }^{\circ} \mathrm{C}$ with primary antibodies against $\beta$-Actin, phospho-S6 (240/244), S6, phospho-EGFR (Y1068;Cell Signaling Technology, Beverly, MA), and human EGFR (R \& D Systems, Minneapolis, MN). Subsequently, the membranes were washed thrice again, and incubated for 1 hour at room temperature with species-specific horseradish peroxidaseconjugated secondary antibodies. Immunoreactive bands were visualized with SuperSignal West Dura Extended Duration Substrate, an enhanced chemiluminescent substrate (Pierce Biotechnology, Rockford, IL). Each experiment was performed at least three times, independently.

\section{Tumor cell inoculation in severe combined immunodeficiency (SHO-Prkde ${ }^{\text {scid }} \mathrm{Hr}^{\text {hr }}$ ) mice}

We used 6-week-old female SHO-Prkdc ${ }^{\text {scid }} \mathrm{Hr}^{\text {hr }}$ mice (SHO-SCID mice: Charles River, Yokohama, Japan) for the study. For the subcutaneous tumor model, cultured tumor cells (PC-9/ffluc; $3 \times 10^{6}$ cells $/ 0.1 \mathrm{ml}$ ) were implanted subcutaneously into the flanks of each mouse. For the leptomeningeal metastasis model, the scalp was sterilized with $70 \%$ ethanol, and the cultured tumor cells were injected into the leptomeningeal space (between the external occipital protuberance and first cervical vertebra) with a $27 \mathrm{G}$ needle.

The size of subcutaneous tumors and body weights of the mice were measured twice per week, and tumor volume was calculated in $\mathrm{mm}^{3}\left(\right.$ width $^{2} \times$ length/2). After 8 days, the mice were either left untreated or treated orally with gefitinib, erlotinib, afatinib, or AZD9291.

This study was carried out in strict accordance with the recommendations of the Guide for the Care and Use of Laboratory Animals by the Ministry of Education, Culture, Sports, Science, and Technology in Japan. The protocol was approved by the Committee on the Ethics of Experimental Animals and the Advanced Science Research Center, Kanazawa University, Kanazawa, Japan (approval no. AP-153499). All surgeries were performed on mice anesthetized with sodium pentobarbital, and efforts were made to minimize the suffering of the animals.

\section{Luciferase expression and radiographic analyses with the IVIS imaging system}

After inoculation, the quantity of tumors was tracked in live mice by repeated noninvasive optical imaging of tumor-specific luciferase activity using the IVIS Lumina XR Imaging System (PerkinElmer, Alameda, CA). Twenty minutes after an intraperitoneal injection of the luciferase substrate luciferin $(150 \mathrm{mg} / \mathrm{kg})$, the mice were photographed under bright-field illumination and images were overlaid with luminescence data gathered over the 
maximum exposure period (5-30 seconds) in anesthetized by $2 \%$ isofluorane. The intensity of the bioluminescence signal was analyzed using Living Image 4.0 (PerkinElmer) by serially quantifying peak photon flux at the selected region of interest (ROI) covering the tumor. This was corrected for total area of ROI and time lapse during which the bioluminescence signals were picked by the CCD camera and expressed as photo/s $/ \mathrm{cm}^{2} / \mathrm{sr}$.

\section{Immunofluorescence}

Mice brains harvested from mice inoculated with PC-9/ffluc cells into the leptomeningeal space were washed with PBS, and tumor cells was collected in a tube. The tubes were centrifuged at $800 \mathrm{rpm}$ for 15 minutes and cell pellets were subsequently analyzed for S6 phosphorylation. Immunofluorescence analysis was performed as described earlier [22]. Briefly, cells on cover slips were fixed in 1\% paraformaldehyde for 15 minutes, followed by treatment with $0.2 \%$ Triton X-100 on ice for 20 minutes. The coverslips were then incubated with rabbit phosphor S6 (240/244) antibody (Cell Signaling) overnight at $4^{\circ} \mathrm{C}$, followed by incubation for 1 hour with Alexa 488-conjugated goat anti-rabbit (Molecular Probes). Confocal microscopy analysis was performed using an Olympus fluorescence microscope (Olympus, Japan).

\section{Immunohistochemistry of skin tissue samples}

Phospho-p44/42 MAPK (Erk1/2, Thr202/Tyr204, Rabbit monoclonal antibody) was purchased from Cell Signaling Technology. Peroxidase-based immunohistochemistry detection system [EnVision+ System-HRP, Rabbit (DAB+)], antibody diluent, and serum-free protein block were purchased from Dako. Formalin-fixed paraffin-embedded tissue skin sections were subjected to antigen retrieval and endogenous peroxidase blocking, and were incubated with primary antibody overnight at $4{ }^{\circ} \mathrm{C}$. Following overnight incubation, slides were rinsed and incubated with a peroxidase-labeled polymer. The tissue sections were then rinsed and stained with 3,3'-diaminobenzidine (DAB) substrate-chromogen and then counterstained with Hematoxylin Gill I (EMD Millipore) and bluing reagent (EMD Millipore).

\section{GRANT SUPPORT}

This work was supported by the following grants: Grants-in-Aid for Cancer Research (S. Yano, 21390256); Scientific Research on Innovative Areas: "Integrative Research on Cancer Microenvironment Network" (S. Yano, 22112010A01); Grant-in-Aid for the Project for Development of Innovative Research on Cancer Therapeutics (P-Direct), from the Ministry of Education, Culture, Sports, Science, and Technology (MEXT) of Japan; and a research grant from AstraZeneca, Chugai and Boehringer Ingelheim.

\section{CONFLICTS OF INTEREST}

Seiji Yano obtained research grants from AstraZeneca, Chugai Pharma, and Boehringer-Ingelheim, and honoraria from Chugai Pharma and Boehringer-Ingelheim.

\section{REFERENCES}

1. Ceresoli GL, Reni M, Chiesa G, Carretta A, Schipani S, Passoni P, Bolognesi A, Zannini P, Villa E. Brain metastases in locally advanced nonsmall cell lung carcinoma after multimodality treatment: risk factors analysis. Cancer. 2002; 95:605-612.

2. Mitsudomi T, Yatabe Y. Mutations of the epidermal growth factor receptor gene and related genes as determinants of epidermal growth factor receptor tyrosine kinase inhibitors sensitivity in lung cancer. Cancer Sci. 2007; 98:1817-1824.

3. Sekine A, Kato T, Hagiwara E, Shinohara T, Komagata T, Iwasawa T, Satoh H, Tamura K, Kasamatsu T, Hayashihara K, Saito T, Takahashi H, Ogura T. Metastatic brain tumors from non-small cell lung cancer with EGFR mutations: distinguishing influence of exon 19 deletion on radiographic features. Lung Cancer. 2012; 77:64-69.

4. Namba Y, Kijima T, Yokota S, Niinaka M, Kawamura S, Iwasaki T, Takeda Y, Kimura H, Okada T, Yamaguchi T, Nakagawa M, Okumura Y, Maeda H, et al. Gefitinib in patients with brain metastases from non-small-cell lung cancer: review of 15 clinical cases. Clin Lung Cancer. 2004; 6:123-128.

5. Zhang J, Yu J, Sun X, Meng X. Epidermal growth factor receptor tyrosine kinase inhibitors in the treatment of central nerve system metastases from non-small cell lung cancer. Cancer Lett. 2014; 351:6-12.

6. Hoffknecht P, Tufman A, Wehler T, Pelzer T, Wiewrodt R, Schütz M, Serke M, Stöhlmacher-Williams J, Märten A, Maria Huber R, Dickgreber NJ. Afatinib Compassionate Use Consortium (ACUC). Efficacy of the irreversible ErbB family blocker afatinib in epidermal growth factor receptor (EGFR) tyrosine kinase inhibitor (TKI)-pretreated nonsmall-cell lung cancer patients with brain metastases or leptomeningeal disease. J Thorac Oncol. 2015; 10:156-163.

7. Heon S, Yeap BY, Britt GJ, Costa DB, Rabin MS, Jackman DM, Johnson BE. Development of central nervous system metastases in patients with advanced non-small cell lung cancer and somatic EGFR mutations treated with gefitinib or erlotinib. Clin Cancer Res. 2010; 16:5873-5882.

8. Omuro AM, Kris MG, Miller VA, Franceschi E, Shah N, Milton DT, Abrey LE. High incidence of disease recurrence in the brain and leptomeninges in patients with nonsmall cell lung carcinoma after response to gefitinib. Cancer. 2005; 103:2344-2348.

9. Eichler AF, Kahle KT, Wang DL, Joshi VA, Willers H, Engelman JA, Lynch TJ, Sequist LV. EGFR mutation status 
and survival after diagnosis of brain metastasis in nonsmall cell lung cancer. Neuro Oncol. 2010; 12:1193-1199.

10. Weickhardt AJ, Scheier B, Burke JM, Gan G, Lu X, Bunn PA Jr, Aisner DL, Gaspar LE, Kavanagh BD, Doebele RC, Camidge DR. Local ablative therapy of oligoprogressive disease prolongs disease control by tyrosine kinase inhibitors in oncogene-addicted non-small-cell lung cancer. J Thorac Oncol. 2012; 7:1807-1814.

11. Jänne PA, Yang JC, Kim DW, Planchard D, Ohe Y, Ramalingam SS, Ahn MJ, Kim SW, Su WC, Horn L, Haggstrom D, Felip E, Kim JH, et al. AZD9291 in EGFR inhibitor-resistant non-small-cell lung cancer. N Engl J Med. 2015; 372:1689-1699.

12. Sequist LV, Soria JC, Goldman JW, Wakelee HA, Gadgeel SM, Varga A, Papadimitrakopoulou V, Solomon BJ, Oxnard GR, Dziadziuszko R, Aisner DL, Doebele RC, Galasso C, et al. Rociletinib in EGFR-mutated non-smallcell lung cancer. N Engl J Med. 2015; 372:1700-1709.

13. Mochizuki S, Soejima K, Shimoda M, Abe H, Sasaki A, Okano HJ, Okano H, Okada Y. Effect of ADAM28 on carcinoma cell metastasis by cleavage of von Willebrand factor. J Natl Cancer Inst. 2012; 104:906-922.

14. Masuda T, Hattori N, Hamada A, Iwamoto H, Ohshimo S, Kanehara M, Ishikawa N, Fujitaka K, Haruta Y, Murai H, Kohno N. Erlotinib efficacy and cerebrospinal fluid concentration in patients with lung adenocarcinoma developing leptomeningeal metastases during gefitinib therapy. Cancer Chemother Pharmacol. 2011; 67:14651469.

15. Finlay MR, Anderton M, Ashton S, Ballard P, Bethel PA, Box MR, Bradbury RH, Brown SJ, Butterworth S, Campbell A, Chorley C, Colclough N, Cross DA, et al. Discovery of a potent and selective EGFR inhibitor (AZD9291) of both sensitizing and T790M resistance mutations that spares the wild type form of the receptor. J Med Chem. 2014; 57:8249-8267.
16. Togashi Y, Masago K, Masuda S, Mizuno T, Fukudo M, Ikemi Y, Sakamori Y, Nagai H, Kim YH, Katsura T, Mishima M. Cerebrospinal fluid concentration of gefitinib and erlotinib in patients with non-small cell lung cancer. Cancer Chemother Pharmacol. 2012; 70:399-405.

17. Zhao J, Chen M, Zhong W, Zhang L, Li L, Xiao Y, Nie L, $\mathrm{Hu}$ P, Wang M. Cerebrospinal fluid concentrations of gefitinib in patients with lung adenocarcinoma. Clin Lung Cancer. 2013; 14:188-193.

18. Grommes C, Oxnard GR, Kris MG, Miller VA, Pao W, Holodny AI, Clarke JL, Lassman AB. "Pulsatile" high-dose weekly erlotinib for CNS metastases from EGFR mutant nonsmall cell lung cancer. Neuro Oncol. 2011; 13:1364-1369.

19. Milton DT, Azzoli CG, Heelan RT, Venkatraman E, GomezJE, Kris MG, Krug LM, Pao W, Rizvi NA, Dunne M, Miller VA. A phase I/II study of weekly high-dose erlotinib in previously treated patients with nonsmall cell lung cancer. Cancer. 2006; 107:1034-1041.

20. Kawamura T, Hata A, Takeshita J, Fujita S, Hayashi M, Tomii K, Katakami N. High-dose erlotinib for refractory leptomeningeal metastases after failure of standarddose EGFR-TKIs. Cancer Chemother Pharmacol. 2015; 75:1261-1266.

21. Green LM, Reade JL, Ware CF. Rapid colorimetric assay for cell viability: application to the quantitation of cytotoxic and growth inhibitory lymphokines. J Immunol Methods. 1984; 70:257-268.

22. Ebi H, Matsuo K, Sugito N, Suzuki M, Osada H, Tajima K Ueda R, Takahashi T. Novel NBS1 heterozygous germ line mutation results in loss of the MRE11-binding domain and increases the predisposition to common types of cancer. Cancer Res. 2007; 67:11158-11165. 